\title{
A Fault Diagnosis Method Based on CFPN Model
}

\author{
Qiumin Fan ${ }^{1, a^{*}}$, Bing $X u^{2, b}$ and Ziqing Chai ${ }^{3, c}$
}

\begin{abstract}
${ }^{1}$ Shanghai Institute of Technology(Haiquan Road N.100,Fengxian District,Shanghai), China
${ }^{2}$ Shanghai Institute of Technology(Haiquan Road N.100,Fengxian District,Shanghai), China ${ }^{3}$ Beijing Institute of Technology(Zhongguancun South Street N.5,Haidian District,Beijing), China

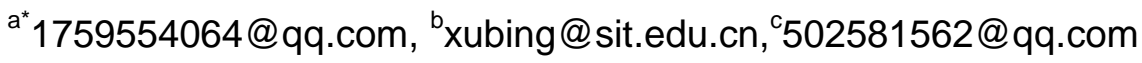

\section{Keywords: CFPN;fault diagnosis;distillation column}

\begin{abstract}
In traditional FPN model,the matrix operations are too excessive and it does not meet the requirements of real-time in fault diagnosis system. The paper has improved the FPN model and proposed a CFPN (Controlled Fuzzy Petri Net) modeling method.This method has added controlled place in FPN and improved the design method of control arc of the control place.The method is based on the principle of p- and it enables to simplify the logic of FPN fault diagnosis. That can reduce the matrix computation in FPN fault diagnosis reasoning to improve the efficiency of fault diagnosis. This paper has applied the method in the distillation column fault diagnosis system and verified the applicability and effectiveness of fault diagnosis method based on CFPN model.
\end{abstract}

\section{Introduction}

FPN is a pretty good modeling tool based on fuzzy production rules knowledge base system because it has fuzzy reasoning ability and it is widely used in the areas of artificial intelligence, fault diagnosis, knowledge representation and so on.About the research of FPN, many scholars have been improved the performance.Such as:combined FPN and BP net ${ }^{[1]}$,apply the DE algorithm in $\mathrm{FPN}^{[2]}$,improved dynamic adaptive $\mathrm{FPN}^{[3]}$ and so on. These improved methods has improved the real-time performance of FPN,but for complex systems, the efficiency is too low.CFPN proposed in this paper has added control arc in FPN and it greatly simplifies the matrix operations of complex system FPN and improves the efficiency of FPN.

So far, the design method for control arc is in continuous improvement.Identification and Parikh mixed constraint proposed by Iordache is not take into account the problem of uncontrollable transitions in Petri net ${ }^{[4]}$.In reference [5],it uses state equation of Petri net to convert the mixed constraint of identification vector and transition vector to the inequality constraints of transition vector. This method is simple, but will lead to deadlock.Based on the existing problems of control arc design,this paper has improved the design method of control arc.And has proposed the efficient control arc design method that in the case of there are uncontrollable transitions in system and has transition identification and transition firing mixed constraint.This paper has combined the method with FPN and proposed a fault diagnosis method based on CFPN model.And it has simplified the 
FPN fault diagnosis model and enhanced the real-time performance of FPN.Finally,the paper has applied CFPN to the distillation column fault diagnosis system to verify the applicability and effectiveness of CFPN.

\section{The Implementation of CFPN}

\subsection{Linear Inequality Constraints}

In FPN control system,linear inequality constraints usually refers to a simple identity constraint and it has the following form:

$$
L \mu \leq b
$$

Among them, $L \in Z^{n_{c} \times n}, \mu \in Z^{n \times 1}, b \in Z^{n_{c} \times 1}, L$ is coefficient matrix, $\mu$ is correlation matrix,b is a positive integer. $n$ is the number of places in FPN, $n_{c}$ is the number of inequality constraints.Identification vector inequality constraints have a very strong modeling capabilities,formula (1) usually used to constrain the prohibition of state.

More general linear inequality constraints are as follows:

$$
C=L_{r} \mu_{r}+\lambda_{r} q_{r} \leq b
$$

Among them, $q_{r}$ is excitation index of the $\mathrm{i}$-th transition. $q_{r}=1$ means the $\mathrm{r}$-th transition is excited. $\lambda_{r} \in Z^{n_{c} \times n_{r}}, n_{r}$ is the number of constraint transitions. $n_{c}$ is the number of constraints, $L_{r}$ is coefficient matrix, $\mu_{r}$ is correlation matrix.Inequality constraints of the form of formula (2) is called place capacity and transitions excited mixed constraint.As can be seen that formula (2) is able to describe the number of implementation of constraint transitions. Thus,compared with the formula (1),formula (2) has stronger description ability.

\subsection{Constraint Conversion Method}

If a transition implementation can not be disabled by an external behavior,then the transition is called uncontrollable transition. The implementation or not of an uncontrollable transition can only be determined by the structure and status of CFPN and regardless of the external environment. When given a set of constraints and there are uncontrollable transitions in FPN system,calculate control arc directly will lead to the occurrence of not allowed constraints ${ }^{[6]}$. Before design the control arc directly,the not allowed constraints need to be converted to allowed constraints.

The steps of mixed constraint conversion algorithm of formula (2) are as follows:

Step 1 The formula (2) is equivalent transformation into:

$$
\begin{aligned}
& L_{r} \mu_{r} \leq k \\
& \lambda_{r} q_{r} \leq b-k
\end{aligned}
$$

Among them, $k \in[0, b]$ 
Step 2 Formula (3) is processed as follows:determine whether to allow constraints. That means whether it meets $L D_{u c} \leq 0$.Among them, $D_{u c}$ is the sub-matrix associated with uncontrollable transitions association matrix ${ }^{[7]}$.If it is, it does not need to be processed,otherwise, go to step 3 .

Step 3 If $L D_{u c}>0$, it is not the allowed constraint,formula (3) needs to be converted to allowed constraint:

$$
L^{\prime} \mu_{r} \leq k
$$

Theorem:Make $R \in Z^{n_{c} \times(k+h)}$,for any $\mu_{r}$, meet $R \mu_{r} \geq 0$, among them, $\mu_{r}$ is the constraint transition identification; If $L_{r}^{\prime} \mu_{r} \leq b$, then $L_{r} \mu_{r} \leq b$.Among them, $L_{r}^{\prime}=R+L_{r}{ }^{[8]}$.

Step 4 Adding formula (4) and (5),get a new allowed constraint:

$$
C^{\prime}=L_{r}^{\prime} \mu_{r}+\lambda_{r} q_{r} \leq b
$$

Among them, $L_{r}^{\prime}$ is determined by the methods in references ${ }^{[9]}$.According to the new constraints of formula (6),the legal controlled arc can be get and it meets the uncontrollable guidelines.

\subsection{Control Arc Design}

This paper uses p- invariant method to design the control $\operatorname{arc}^{[10]}$ and considers including the exist of place capacity and transition firing mixed constraints and uncontrollable transitions.For ease of discussion, assume that:

(1)FPN model has no loopback;

(2)Constraint transitions are all considerable and controllable.

The mixed constraint is expressed as $\mathrm{C}$ :

$$
C=\left\{\sum_{r=1}^{n} l_{r} \mu_{r}+\sum_{r=1}^{n} \lambda_{r} q_{r} \leq b\right\}
$$

Among them,places and transitions are called constraint places and constraint transitions.

Control arc design steps are as follows:

Step 1 Determine the constraint place $C_{p}$ and constraint transition $C_{t}$.

Step 2 According to the constraints, write local incidence matrix of constraint place:

$$
D_{0}=\underset{p_{1}}{p_{1}}\left[\begin{array}{cccc}
d_{11} & d_{2} & \cdots & t_{j} \\
p_{k} & \cdots & d_{1 j} \\
\vdots & \vdots & & \vdots \\
d_{k 1} & d_{k 2} & \cdots & d_{k j}
\end{array}\right]
$$

In formula (8), $p_{1}, p_{2}, \cdots, p_{k}$ is constraint places, $t_{1}, t_{2}, \cdots, t_{j}$ is transitions and constraint transitions associated with the constraint places.

It can be seen from formula (8),the numbers of dimensions and columns of local incidence matrix are all less than or equal to the FPN correlation matrix.And it will not increase when the size of the system becomes large.It allows the design complexity of control arc greatly reduced.At the same time,for complex systems, it will be largely simplified.

Step 3 According to constraints, write the coefficient matrix of constraint places: 


$$
L_{r}=\left[\begin{array}{cccc}
l_{11} & l_{12} & \cdots & l_{1 k} \\
\vdots & \vdots & & \vdots \\
l_{n_{c} 1} & l_{n_{c} 2} & \cdots & l_{n_{c} k}
\end{array}\right]
$$

Step 4 Determine the permissibility of constraints.That means whether it meets $L D_{u c} \leq 0$, if satisfied,it is allowed constraint.Then make $L_{r}^{\prime}=L_{r}$ and go to step 6,or go to step 5 .

Step 5 Convert not allowed constraint to allowed constraint and get the new allowed constraint:

$$
L_{r}^{\prime} \mu_{r}+\lambda_{r} q_{r} \leq b
$$

Step 6 According to the local incidence matrix $D$ and coefficient matrix $L_{r}^{\prime}$ of constraint place,combined with FPN simplifying technology,the local incidence matrix of control places can be showed as $D_{c}=-L_{r}^{\prime} D$

Step 7 Add coefficient transition row $t_{r}$ for the local incidence matrix of control places $D_{c}$. Fill 1 in the positions of corresponding constraint transitions in $t_{r}$ row and fill 0 in other positions. That is:

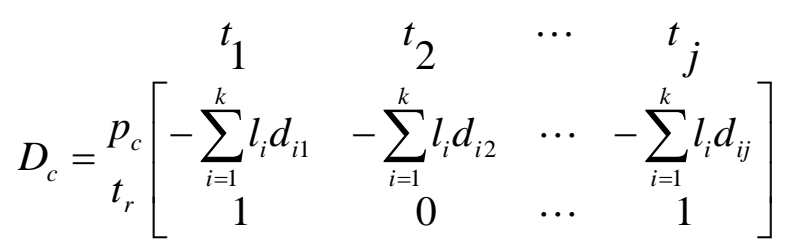

Consider the distribution of $D_{c}$ elements,construct the corresponding arc according to the following rules ${ }^{[11]}$ :

(1)The number of constraints is equal to the number of constraint places;

(2)If the corresponding matrix element of the last line element 1 is 0 ,then construct a two-way arc between $p_{c}$ and transition.Make $t$ are both an input transition and an output transition of $p_{c}$. This makes $p_{c}$ become common input place of all the constraint transitions and satisfies the constraint inequality.

(3)If an element that the in the last line element 1 corresponding matrix is less than 0 ,then there is no arc between $t$ and $p_{c}$.

(4)If the the last line element 1 and 0 corresponding element in matrix is more than 0 and two transitions related has common input place,construct a two-way arc between control places and this transition.Otherwise, this method can not be used to design control arc because control arc may introduce deadlocks.

(5)When a transition excitation leads to the increase of identification in constraints, then make this transition become an output of control place,or an input.The corresponding arc weight is equal to the number of identity that increase or decrease.

Step 8 Calculate the initial identification of control place:

$$
\mu_{c_{0}}=b-L_{r}^{\prime} \mu_{r 0}
$$

Among them, $\mu_{r 0}$ is the matrix consists of the number of initial tokens of constraint place.Based on the above principles, add the designed control arc to FPN model,then that is the CFPN model.

\section{CFPN Modeling Method in Distillation Column Fault Diagnosis}

Distillation column has complex system structure and has more uncertainties,so it has the larger probability of failure.Traditional fault diagnosis methods can not meet the requirements.The CFPN model proposed by this paper is suitable for the system that has complex structure and large matrix operations.Therefore, it can be used to the fault diagnosis of distillation column.For the distillation 
column body fault,this paper has established its CFPN model and then do fault reasoning.It can do fault diagnosis quickly and accurately.

\subsection{Fault FPN Model}

We classify the common fault of distillation tower body and sum up the common fault of distillation tower body with the corresponding failure symptoms.Specifically as shown in Table I :

Table I Common faults of distillation tower body and fault symptom correspondence table

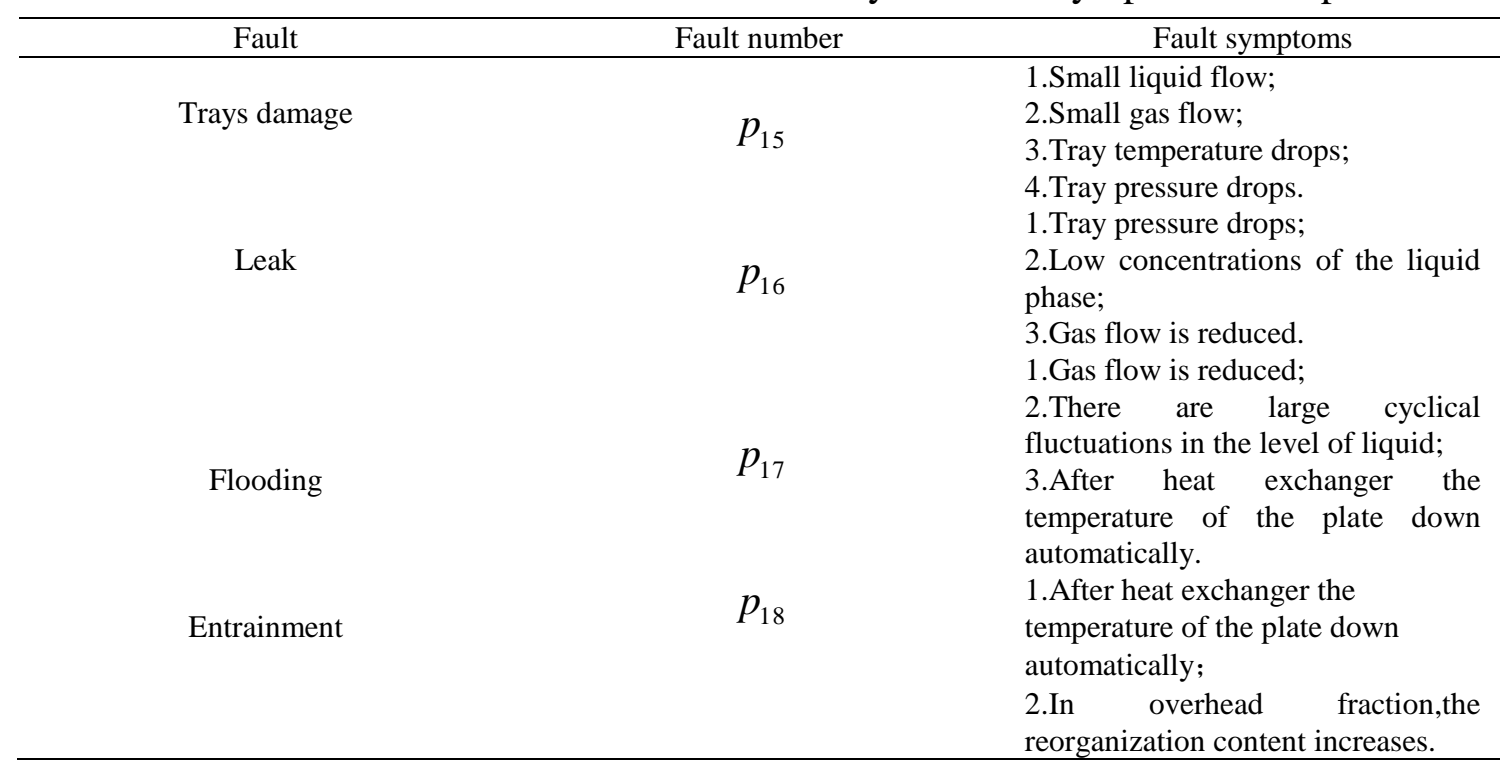

The FPN uncertain knowledge representation rules of common faults of distillation tower body are described below:

Rule 1 if $p_{1}$ and $p_{2}$ and $p_{3}$ and $p_{4}$ then $p_{10}\left(\lambda_{1} 、 \mu_{1} 、 \omega_{1} 、 \omega_{2} 、 \omega_{3} 、 \omega_{4}\right)$;

Rule 2 if $p_{4}$ and $p_{5}$ and $p_{6}$ then $p_{11}\left(\lambda_{2} 、 \mu_{2} 、 \omega_{5} 、 \omega_{6} 、 \omega_{7}\right)$;

Rule 3 if $p_{6}$ and $p_{7}$ then $p_{12}\left(\lambda_{3} 、 \mu_{3} 、 \omega_{8} 、 \omega_{9}\right)$;

Rule 4 if $p_{8}$ then $p_{13}\left(\lambda_{4} 、 \mu_{4}\right)$;

Rule 5 if $p_{8}$ and $p_{9}$ then $p_{14}\left(\lambda_{5} 、 \mu_{5} 、 \omega_{10} 、 \omega_{11}\right)$;

Rule 6 if $p_{10}$ then $p_{15}\left(\lambda_{6}, \mu_{6}\right)$;

Rule 7 if $p_{11}$ then $p_{16}\left(\lambda_{7} 、 \mu_{7}\right)$;

Rule 8 if $p_{12}$ and $p_{13}$ then $p_{17}\left(\lambda_{8} 、 \mu_{8} 、 \omega_{12} 、 \omega_{13}\right)$;

Rule 9 if $p_{14}$ then $p_{18}\left(\lambda_{9}, \mu_{9}\right)$.

Among them, $\omega_{j} 、 \mu_{i} 、 \lambda_{k}(j, i, k \in N)$ represent the weights, certainty and threshold.Each place is defined as shown in the following table: 
Table II Place definition table

\begin{tabular}{|c|c|c|c|c|c|}
\hline Place & Meaning & Place & Meaning & Place & Meaning \\
\hline$P_{1}$ & $\begin{array}{l}\text { Small liquid } \\
\text { flow }\end{array}$ & $P_{7}$ & $\begin{array}{c}\text { There are } \\
\text { large } \\
\text { cyclical } \\
\text { fluctuations } \\
\text { in the level } \\
\text { of liquid }\end{array}$ & $P_{13}$ & $\begin{array}{l}\text { Each tray } \\
\text { pressure } \\
\text { drops }\end{array}$ \\
\hline$P_{2}$ & Small gas flow & $P_{8}$ & $\begin{array}{l}\text { After heat } \\
\text { exchanger the } \\
\text { temperature } \\
\text { of the plate } \\
\text { down } \\
\text { automatically }\end{array}$ & $P_{14}$ & $\begin{array}{l}\text { Gas samples } \\
\text { produced from } \\
\text { overhead with } \\
\text { a clear liquid } \\
\text { phenomenon }\end{array}$ \\
\hline$P_{3}$ & $\begin{array}{c}\text { Tray } \\
\text { temperature } \\
\text { decreases }\end{array}$ & $P_{9}$ & $\begin{array}{l}\text { In overhead } \\
\text { fraction,the } \\
\text { reorganization } \\
\text { content } \\
\text { increases }\end{array}$ & $p_{15}$ & Trays damage \\
\hline$P_{4}$ & $\begin{array}{c}\text { Tray pressure } \\
\text { drop }\end{array}$ & $P_{10}$ & $\begin{array}{l}\text { Low tray } \\
\text { efficiency }\end{array}$ & $p_{16}$ & Leak \\
\hline$P_{5}$ & $\begin{array}{c}\text { Low } \\
\text { concentration } \\
\mathrm{s} \text { of the } \\
\text { liquid phase }\end{array}$ & $P_{11}$ & $\begin{array}{l}\text { Each segment } \\
\text { resistance } \\
\text { decrease } \\
\text { along tower }\end{array}$ & $p_{17}$ & Flooding \\
\hline$P_{6}$ & $\begin{array}{l}\text { Gas flow is } \\
\text { reduced }\end{array}$ & $P_{12}$ & $\begin{array}{l}\text { High, medium } \\
\text { pressure drop } \\
\text { in tower }\end{array}$ & $p_{18}$ & Entrainment \\
\hline
\end{tabular}

Based on the FPN uncertain knowledge representation rules of common faults of distillation tower body,to establish its FPN uncertain knowledge representation model.As shown in Fig. 1:

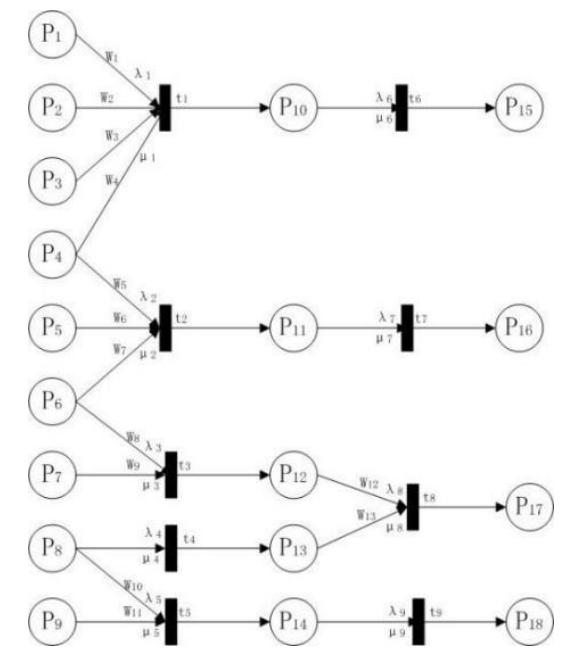

Figure 1: Uncertainty knowledge representation FPN model of distillation tower

\subsection{The Control Arc Design of Fault CFPN Model}

According to the control arc design method in this paper,suppose the mixing places and transitions constraints need to achieve is as follows ${ }^{[12]}$.

$$
\mu_{8}+\mu_{9}+q_{4}+q_{5} \leq 4
$$

Among them, $t_{3}$ is the uncontrollable transition.

Control arc design process is as follows:

Firstly,construct $D$ and $L_{r}$. 


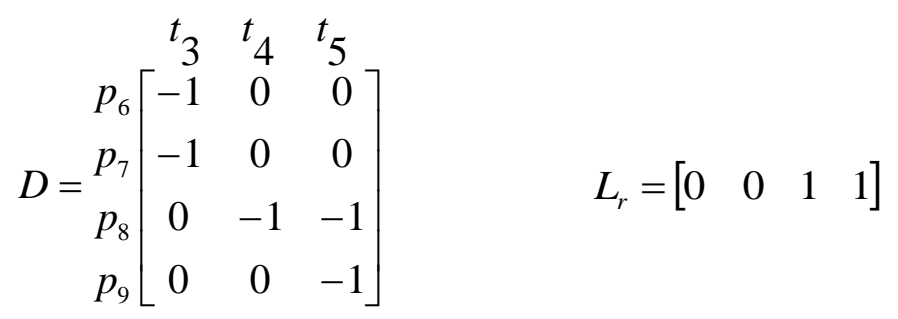

It is noteworthy that except $t_{3}, t_{4}, t_{5}$, all transitions are both unrelated with constraint places and constraint transitions and unrelated with uncontrollable transitions. So the $D$ does not include other transitions and it reduces the complexity of matrix operations. When the system has large-scale and higher incidence matrix dimension, the advantages of this method of calculation is more obvious.

Because $t_{3}$ is the uncontrollable transition, $D_{u c}=\left[\begin{array}{c}-1 \\ -1 \\ 0 \\ 0\end{array}\right]$.

Determine the constraints permissibility.Calculate $L_{r} D_{u c}=0$, meet the allowed constraints,so there is no need to do constrain conversion and $L_{r}^{\prime}=L_{r}$. Then obtained a local incidence matrix for the control place:

$$
D_{C}=-L_{r}^{\prime} D=\left[\begin{array}{lll}
0 & 1 & 2
\end{array}\right]
$$

Then according to step 7:

$$
D_{c}=p_{t_{r}}\left[\begin{array}{lll}
0 & 1 & 2 \\
0 & 1 & 1
\end{array}\right]
$$

Finally, calculate the initial identification of control place:

$$
\mu_{0}=b-L_{r}^{\prime} \mu_{r 0}=4-\left[\begin{array}{llll}
0 & 0 & 1 & 1
\end{array}\right]\left[\begin{array}{l}
2 \\
1 \\
2 \\
1
\end{array}\right]=1
$$

According to the control arc con $\left.\mathrm{P}_{2}\right) \rightarrow$ model is as Fig. 2:

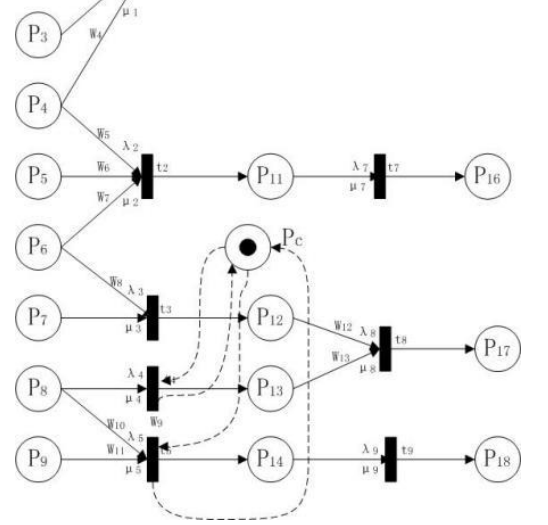

Figure 2:Distillation column fault CFPN model

In figure 2, the dotted line is the arc between control arc and related transitions.It can be seen the participation of control arc greatly simplifies FPN model.For the distillation tower body faults, use MATLAB to simulate.Simulate 10 times for the matrix operations speed and the accuracy of fault diagnosis of different fault based on FPN model and CFPN model.The comparison of averages are shown in Table III: 
Table III Matrix computation time and accuracy of two fault diagnosis model

\begin{tabular}{|c|c|c|c|c|}
\hline \multirow{2}{*}{ Fault } & \multicolumn{2}{|c|}{ Time (average value) $/ \mathrm{ms}$} & \multicolumn{2}{c|}{ Accuracy (average value) } \\
\cline { 2 - 5 } & FPN & CFPN & FPN & CFPN \\
\hline Trays damage & 5.22 & 1.70 & 0.78 & 0.95 \\
\hline Leak & 6.17 & 3.58 & 0.81 & 0.92 \\
\hline Flooding & 4.92 & 1.82 & 0.72 & 0.94 \\
\hline Entrainment & 3.84 & 1.61 & 0.89 & 0.96 \\
\hline
\end{tabular}

As can be seen from table III,CFPN fault diagnosis efficiency is superior to FPN and the real-time performance of CFPN is improved.

\section{Conclusions}

This paper has improved the FPN fault diagnosis method.The paper has simplified the FPN model and has improved the real-time performance of FPN by add control arc.In the process of designing control arc,using p- invariant approach and the local incidence matrix algorithms to reduce the incidence matrix dimension to reduce the calculation of FPN.Finally,For the numbers of complex faults in distillation tower body,apply CFPN to the distillation tower body fault diagnosis and verify the applicability and effectiveness of CFPN.

\section{References}

[1]E. Salimi; R. S. Sreenivas, On Invariant-Based Monitors That Enforce Liveness in a Class of Partially Controlled General Petri Nets[J].IEEE Journals \& Magazines,2015,60 (89-92) .

[2]X. Wang; Z. Li; W. M. Wonham, Dynamic Multiple-Period Reconfiguration of Real-Time Scheduling Based on Timed DES Supervisory Control[J],IEEE Journals \& Magazines,2016,12(21-26).

[3]M. Teixeira; R. Malik; J. E. R. Cury; M. H. de Queiroz, Supervisory Control of DES With Extended Finite-State Machines and Variable Abstraction[J],IEEE Journals \& Magazines,2015,60(06-10).

[4]J. Luo; H. Ni; W. Wu; S. Wang; M. Zhou,Simultaneous Reduction of Petri Nets and Linear Constraints for Efficient Supervisor Synthesis[J],IEEE Journals \& Magazines,2015,60(51-60).

[5]Leitão,H.A.S.,Lopes,W.T.A.,Madeiro,F.,PSO Algorithm Applied to Codebook Design for Channel-Optimized Vector Quantization[J],Latin America Transactions, IEEE (Revista IEEE America Latina),2015,13(78-82).

[6]Silva,E.H.M.,Bastos Filho,C.J.A.PSO Efficient Implementation on GPUs Using Low Latency Memory[J].Latin America Transactions,IEEE (Revista IEEE America Latina),2015,13(05-09)..

[7]Samani,M.,Tafreshi,M.,Shafieenejad,I.,Nikkhah,A.A..Minimum-time open-loop and closed-loop optimal guidance with GA-PSO and neural fuzzy for Samarai MAV flight[J].Aerospace and Electronic Systems Magazine,IEEE,2015,30(33-38).

[8]Fei,R.,Moreau,J.,Mir,S.,Marcellin,A.,Mandier,C.;Huss,E.,Palmigiani,G.,Vitrou,P.,Droniou,T..Ho rizontal-FPN fault coverage improvement in production test of CMOS imagers[J].VLSI Test Symposium (VTS),IEEE,2015,33(121-125).

[9]Chen,S.-J.,Zhan,T.-S.,Huang,C.-H.,Chen,J.-L.,Lin,C.-H..Nontechnical Loss and Outage Detection Using Fractional-Order Self-Synchronization Error-Based Fuzzy Petri Nets in Micro-Distribution Systems[J].Smart Grid,IEEE Transactions on,2015,06(64-69). 
[10]Wei-Ling Chen,Chung-Dann Kan,Chia-Hung Lin,Tainsong Chen,A Rule-Based Decision-Making Diagnosis System to Evaluate Arteriovenous Shunt Stenosis for Hemodialysis Treatment of Patients Using Fuzzy Petri Nets[J].Biomedical and Health Informatics,IEEE Journal of,2014,18(45-51).

[11]Gniewek,L.,Coverability Graph of Fuzzy Interpreted Petri Net[J].Systems, Man, and Cybernetics:Systems,IEEETransactions on,2014,44(38-42).

[12]L. Aggoune; Y. Chetouani; H. Radjeai, Change detection in a distillation column using non-linear auto-regressive moving average with exogenous input model and Hellinger distance[J],IET Journals \& Magazines,2016,10(11-16). 